\title{
New Neutron Cross Section and Fission Yield Data for SNM Analysis
}

\author{
R.B. Firestone (rbf@lbl.gov) \\ Lawrence Berkeley National Laboratory \\ G.L. Molnar, T. Belgya, and Zs. Revay \\ Institute of Isotope and Surface Chemistry, Budapest, Hungary
}

Operational Capability: Neutron cross-section data are fundamental for the design of nuclear interrogation systems, the maintenance of nuclear materials and waste, and the understanding the consequences of nuclear catastrophe. Although a large body of nuclear data exists, it is often old, unreliable, or poorly determined. For several years we have collaborated, as part of an IAEA Coordinated Research Project, to precisely measure the partial thermal neutron gamma ray cross sections for all elements from hydrogen to uranium at the Budapest Reactor. These data will replace the unreliable tables of Lone et al [1], still widely in use, and will be published as an IAEA TECDOC.

A byproduct of this effort is the determination of precise total isotopic cross sections for most isotopes. A summary of our results for selected light elements is shown in Table 1. The cross sections for neutron capture on ${ }^{6} \mathrm{Li}$ and ${ }^{12} \mathrm{C}$ disagree significantly with the compilation by Mughaghab et al [2] and may be critical for reactor calculations. Many other discrepancies have been found for heavier elements. Dr. Mughabghab has agreed to help evaluate these new results.

\begin{tabular}{|c|c|c|c|c|}
\hline Isotope & $\sigma$ (Literature) & $\sigma$ (this work) & \multicolumn{2}{|c|}{ Isotope \%Yield(England) \%Yield(this work) } \\
\hline${ }^{1} \mathrm{H}$ & $332.6 \mathrm{mb} 7$ & $332.6 \mathrm{mb}$ & ${ }^{100} \mathrm{Zr} \quad 5.4$ & $5.1(5)$ \\
\hline${ }^{2} \mathrm{H}$ & $0.519 \mathrm{mb} 7$ & $0.492 \mathrm{mb} 25$ & ${ }^{134} \mathrm{Te}$ & $4.9(1)$ \\
\hline${ }^{6} \mathrm{Li}$ & $39 \mathrm{mb} 3$ & $52.6 \mathrm{mb} 22$ & ${ }^{138} \mathrm{Xe}$ & $5.1(4)$ \\
\hline${ }^{7} \mathrm{Li}$ & $45 \mathrm{mb} 3$ & $45.7 \mathrm{mb} 9$ & \multirow{3}{*}{\multicolumn{2}{|c|}{$\begin{array}{l}\text { Table 2. Comparison of }{ }^{23 b} \mathrm{U} \text { literature fission } \\
\text { yields with preliminary results measured at } \\
\text { the Budapest Reactor. }\end{array}$}} \\
\hline${ }^{9} \mathrm{Be}$ & $7.6 \mathrm{mb} 8$ & $8.8 \mathrm{mb} 6$ & & \\
\hline${ }^{10} \mathrm{~B}$ & $500 \mathrm{mb} 200$ & $303 \mathrm{mb} 20$ & & \\
\hline${ }^{10} \mathrm{~B}(\mathrm{n}, \alpha)$ & 712.5 b 17 & $712.5 \mathrm{~b}$ & & \\
\hline${ }^{12} \mathrm{C}$ & $3.53 \mathrm{mb} 7$ & $3.89 \mathrm{mb} 6$ & & \\
\hline${ }^{13} \mathrm{C}$ & $1.37 \mathrm{mb} 4$ & $1.22 \mathrm{mb} 11$ & \multirow{4}{*}{\multicolumn{2}{|c|}{$\begin{array}{l}\text { Table 1. Total thermal neutron cross sections } \\
\text { measured at the Budapest Reactor. Additional valu } \\
\text { for all elements up to uranium are still being evaluat }\end{array}$}} \\
\hline${ }^{14} \mathrm{~N}$ & $75 \mathrm{mb} 8$ & $79.0 \mathrm{mb} 9$ & & \\
\hline${ }^{16} \mathrm{O}$ & $0.190 \mathrm{mb} 19$ & $0.189 \mathrm{mb} 8$ & & \\
\hline${ }^{19} \mathrm{~F}$ & $9.6 \mathrm{mb} 5$ & $9.50 \mathrm{mb} 11$ & & \\
\hline
\end{tabular}

Thermal neutron-induced fission yield data are sparse, but necessary for analyzing fissile materials. These data are typically provided by the semi-empirical database of England and Rider [3]. England and Rider were constrained to produce a database that conserves mass for nuclear reactor calculations and had to rely on theory or assumptions to get many values. The quantitative accuracy of their results is questionable. Preliminary fission yield values, from neutron-induced fission on ${ }^{235} \mathrm{U}$ measured at Budapest are shown in Table 2 . The yield of ${ }^{134} \mathrm{Te}$ appears to be discrepant by $20 \%$.

The experimental facilities at the Budapest Reactor are shown in Figure 1. The $5 \times 10^{7} \mathrm{n} / \mathrm{cm}^{2} \mathrm{~s}$ cold neutron beam is uniquely suited for precise measurements of partial gamma-ray and total isotopic thermal neutron cross sections and fission yields for all elements including to the actinides. Development of a more reliable neutron cross-section database will benefit all users who do calculations with nuclear data for nuclear engineering, criticality studies, nuclear nonproliferation, neutron interrogation technologies, and materials research. They also provide a wealth of fundamental new information to nuclear scientists and astrophysics about the nuclear structure and decay of neutron rich nuclei. 


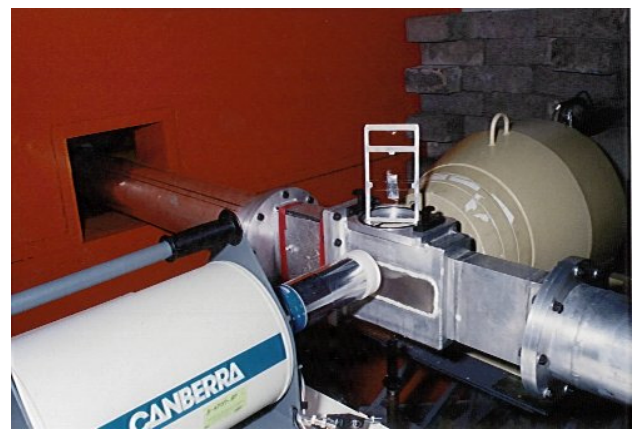

Figure 1. The Budapest Reactor cold neutron beam experimental facility. A 25\% HPGe detector (foreground) is shown pulled away from its Compton suppression system (background). The target assembly is shown in its external position. The $5 \times 10^{7} \mathrm{n} / \mathrm{cm}^{2} \mathrm{~s}$ neutron beam arrives from the right in the neutron guide. We propose replacing the current detector with 2 clover detectors that can achieve high coincidence efficiency in a close geometry and do not require Compton suppression because of their photon add-back capability.

Technical Approach: The unparalleled precision of the Budapest cross section measurements was achieved by measuring the gamma-ray spectra for compounds with well-known stoichiometry where one of the components is a cross section standard, preferably hydrogen or nitrogen. In some cases the secondary standard chlorine was used. Internal standardization directly provides the cross section for the element of interest without requiring corrections for source geometry, neutron flux, or other variables that plagued previous experiments. For most cases several compounds of an element are analyzed to obtain redundant information for quality assurance. Partial gamma ray cross sections are measured directly, and the total cross sections are inferred from total intensity populating an isotopes ground state or, for lighter elements, from the total primary gamma ray intensity.

Special emphasis will go towards measurements of isotopes whose cross sections deviate significantly from Mughaghab's values [2]. Additional separated isotope measurements will be performed to gain more complete information. New data will be measured for the isotopes ${ }^{233} \mathrm{U}$, ${ }^{234} \mathrm{U},{ }^{235} \mathrm{U},{ }^{238} \mathrm{U},{ }^{238} \mathrm{Pu},{ }^{239} \mathrm{Pu},{ }^{240} \mathrm{Pu},{ }^{241} \mathrm{Pu},{ }^{242} \mathrm{Pu}$ and ${ }^{244} \mathrm{Pu}$ on targets available at LBNL. Additional experiments, on isotopes of Americium, and Curium, will be considered to extend the database.

Fission yields will be determined by quantitative gamma-gamma coincidence spectroscopy with an array consisting of two HPGE clover detectors surrounding the target. Each clover contains four, tightly-packed HPGe detectors that can be operated either independently or to add back Compton scattered or annihilation photons, providing the highest possible total peak efficiency. We can obtain 3-fold and greater coincidence data, analogous to that measured with GAMMASPHERE detector at LBNL. This is achieved using closer detector geometry, which is possible due to the lower multiplicity of fission. Detector efficiency will be calibrated to a precision of less than $1 \%$, over the energy range of $0-11 \mathrm{MeV}$, using techniques developed at Budapest. A beam chopper is available to study fission product decays and delineate the independent and cumulative fission yields. The Budapest Reactor has two nearly identical cold neutron beam lines, so data can be acquired continuously for many months without impacting other research. Data will be written to tape in event mode for off-line analysis by conventional techniques, and the coincidence data will be a resource to the broad nuclear research community for future studies.

Cost and Schedule: This proposal requests support for five years of funding commencing in January, 2003 or the start of funding. No support is requested for personnel or beam time in Budapest. Funding for 1 FTE US scientist (\$200K/year) and 1 FTE postdoctoral student ( $\$ 100 \mathrm{~K} /$ year) is requested. Equipment, requested in the first year, includes $2 \mathrm{HPGe}$ clover detectors and associated electronics $(\$ 270 \mathrm{~K})$. We ask additional annual support for travel, computers, isotopes, and supplies ( $\$ 100 \mathrm{~K} / \mathrm{year}$ ). The total request is thus $\$ 670 \mathrm{~K}$ in the first year and $\$ 400 \mathrm{~K}$ for subsequent years. The following project schedule is proposed. 


\section{Project Schedule}

1) Year 1 - Acquire clover detectors, install and calibrate data acquisition systems, continue partial gamma cross-section measurements and evaluate total cross sections.

2) Year 2 - Continue cross section measurements and evaluation and begin measuring fission product yields.

3) Year 3 - Complete cross section evaluation, continue measuring fission product yields, and begin analyzing fission product yield data.

4) Year 4 - Conclude fission product yield measurements and continue analyzing fission product yields.

5) Year 5-Complete evaluation of fission product yields and disseminate all results.

The individuals listed on this proposal, their students, and the proposed postdoctoral candidate will perform all of this work. We will solicit additional collaborators to join the project at their own expense, and expect to expand collaboration.

Deliverables: We will provide a complete and accurate database of thermal neutron crosssections and fission product yields. These data will be published in the open literature and provided to the nuclear data community for incorporation into ENDF/B and the other databases of the US defense laboratories. The evaluated data will be available on the Internet, and the experimental data will be available to qualified users. These data will be made available at no charge, and no commercial exploitation is planned.

[1] M.A. Lone, R.A. Leavitt and D.A. Harrison, Atom. Data Nucl. Data Tables 26, (1981) 511. [2] S.F. Mughabghab, M. Divadeenam, and N.E. Holden, Neutron Cross Sections, Vol. 1: Neutron Resonance Parameters and Thermal Cross Sections, Part A Z=1-60, Academic Press (1981); S.F. Mughabghab, Neutron Cross Sections, Vol. 2: Neutron Resonance Parameters and Thermal Cross Sections\}, Part B Z=61-100, Academic Press (1984).

[3] Evaluation and Compilation of Fission Yields, T.R. England and B.F. Rider, ENDF-349, LAUR-94-3106. 